\title{
EFICACIA DE ANTIPARASITARIOS ORALES EN EL TRATAMIENTOY CONTROL DE NEMATODOS GASTROINTESTINALES EN EQUINOS
}

\section{Efficacy of oral antiparasitic agents in the treatment and control of Gastrointestinal nematodes in horses}

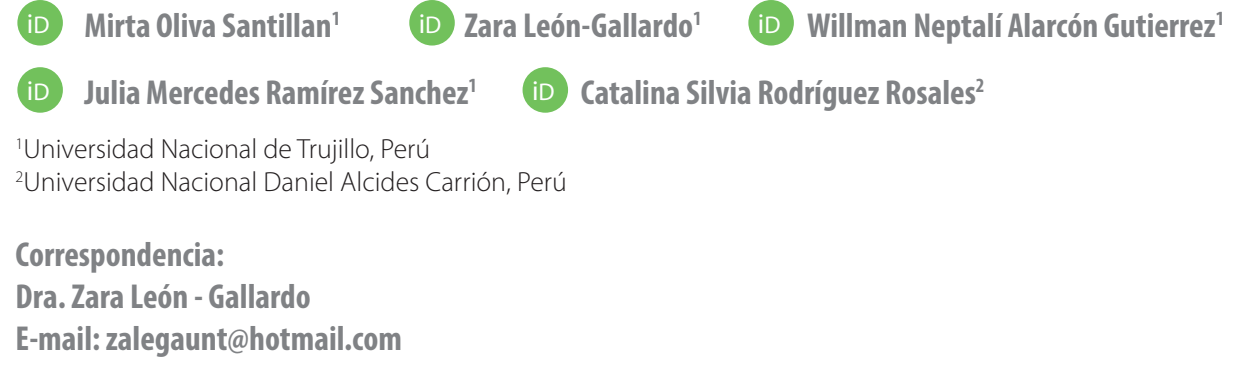

\section{RESUMEN}

El estudio tuvo como objetivo determinar la eficacia de dos antiparasitarios orales en el control de nematodos gastrointestinales en equinos. Se utilizaron 45 yeguas de 2 a 4 años distribuidas aleatoriamente en tres grupos: I: grupo control, II: tratamiento con antiparasitario. doramectina en dosis de $11,4 \mathrm{mg} / \mathrm{kg}$ de peso vivo y III: tratamiento con antiparasitario fenbendazole en dosis de $7,5 \mathrm{mg} / \mathrm{kg}$ de peso vivo, con 15 repeticiones cada uno. Se colectaron muestras de heces previas a la dosificación y a los 30 días post tratamiento. Las muestras fecales fueron analizadas mediante la técnica de Baermann modificada en copa, Kato-Katz y la prueba de Graham. Los datos de pre y post tratamiento se analizaron con pruebas paramétricas y no paramétricas. La eficacia de los tratamientos se determinó mediante el método de porcentaje de reducción de huevos por gramo de heces (hpg). Todas las yeguas resultaron positivas a nematodos, identificándose Strongylus sp, Parascaris spy Oxyuris sp. La eficacia de la doramectina fue de $97 \%$ y del fenbendazole $51 \%(p<0.05)$.

Palabras clave: eficacia antiparasitaria, nematodiasis, yeguas, coproparasitología .

\section{ABSTRACT}

The study aimed to determine the efficacy of two oral antiparasitic drugs in the control of gastrointestinal nematodes in horses. 45 mares from 2 to 4 years old, distributed randomly into three groups: I: control group, II: antiparasitic treatment. doramectin in doses of $11.4 \mathrm{mg} / \mathrm{kg}$ of body weight and III: treatment with antiparasitic fenbendazole in doses of $7.5 \mathrm{mg} / \mathrm{kg}$ of body weight, with 15 repetitions each. Stool samples were collected prior to dosing and 30 days after treatment. The fecal samples were analysed using the modified Baermann technique in cup, Kato-Katz, and the Graham test. Pre and post treatment data were analyzed with parametric and non-parametric tests. The efficacy of the treatments was determined by the method of percentage of reduction of eggs per gram of feces (hpg). All the mares were positive to nematodes, identifying 
Strongylus sp, Parascaris sp and Oxyuris sp. The efficacy of doramectin was $97 \%$ and fenbendazole $51 \%(p<0.05)$.

Key words: antiparasitic efficacy, nematodiasis, mares, coproparasitología.

\section{INTRODUCCIÓN}

Los équidos, como el caballo (Equus caballus), son utilizados para el trabajo en zonas rurales o suburbanas, usualmente reciben poco cuidado, inadecuada alimentación y escasa atención veterinaria (Burn et al., 2009), factores que los predispone a contraer diversos problemas sanitarios, incluyendo las parasitosis (Teixeira et al., 2014; Matto et al., 2015) y, dentro de ellas, la nematodiasis, parasitosis que les generan una serie de trastornos digestivos que afectan el estado físico, absorción de nutrientes y eficiencia alimenticia, ocasionándoles una baja productividad en su desempeño (Gutiérrez y Gutiérrez, 2013).

Entre los nematodos se distinguen tanto los gastrointestinales (NGl) (Rojas, 2004; Nielsen, 2012; Von Samson-Himmelstjerna, 2012) como los grandes y pequeños estróngilos (o ciatostomas) y los oxiuros (Osterman, 2005). Los estróngilos son de ciclo biológico directo y la transmisión es por vía oral cuando los caballos ingieren las larvas infectantes al pastorear (Reinemeyer, 2009). Los pequeños estróngilos y el Parascaris equorum son los parásitos más comunes e importantes del equino (Francisco et al., 2012; Peregrine et al., 2014). Por otro lado, el Oxyuris equies un gusano redondo, cuyas hembras adultas se desplazan del intestino grueso hacia el recto, donde ponen huevos en la región perianal, ocasionando inflamación (Velásquez, 2012; Reinemeyer, 2008). Los parásitos se encuentran contaminando las pasturas. Por ello, mantener una baja carga parasitaria en el pasto es una medida de control importante.

Los parásitos afectan a los equinos en sus diferentes etapas de crecimiento y es casi imposible que se encuentren totalmente libre de éstos. Particularmente es importante el control de las parasitosis en yeguas por su importancia en la reproducción, por constituir éstas, una vía de transmisión de parásitos con la ingestión más frecuente de larvas infectantes a través de la leche materna (Reinemeyer, 2008), así como reducir los riegos de infección percutánea a través de la ingestión de larvas infectantes que se encuentran en el ambiente.

Actualmente, existen tres grupos químicos con acción sobre los nematodos en equinos: bencimidazoles (fenbendazole, oxfenbendazol, oxibendazol), tetrahidropirimidinas (sales de pirantel) y lactonas macrocíclicas (ivermectina, doramectina, moxidectina) (Kaplan, 2005; Matthews, 2008; Reinemeyer, 2009) con efectos variados. La mayoría de estos antiparasitarios son también eficaces contra otros parásitos de los caballos. Las formulaciones orales, por su menor actividad sistémica, son altamente eficaces frente a endoparásitos, pero, no suelen ser eficaces frente a ectoparásitos; este es el caso de las formulaciones para caballos, como lo reporta Wang (2001).

Algunos estudios, como los de Tang y Ledesma (2006), demostraron que la aplicación de un gel endectocida saborizado oral sobre la base de doramectina al 1.75\% (p/p) tuvo 100\% de eficacia en la reducción de huevos tipo Strongylus en heces. Chávez et al., (2007) evidenciaron eficacia de fenbendazole contra nematodos durante los días 7 y 35 post tratamiento de 100 y 90.9\%, respectivamente. Asimismo, Reinemeyer (2008), trabajando con yeguas de preñez avanzada encontró que las lactonas macrocíclicas (ivermectina $0.2 \mathrm{mg} / \mathrm{kg}$ o moxidectina $0.4 \mathrm{mg} /$ kg) bloquearon la transmisión vertical, matando las larvas de Strongyloides en sus reservorios somáticos, antes de la migración y transmisión lactogénica. Chávez (2013), considera que la administración oral de doramectina, por su eficacia antihelmíntica, es una alternativa a los antiparasitarios convencionales para el tratamiento y control de las enfermedades parasitarias en equinos; sin embargo, son muy limitados los antecedentes de su uso en los equinos. 
Por lo antes expuesto y, siendo la doramectina un fármaco de elección por su tiempo de acción, no tóxica y de fácil aplicación, se planteó la presente investigación, teniendo como objetivo determinar la eficacia de dos antiparasitarios orales en el tratamiento y control de nematodos gastrointestinales en equinos hembras de La Libertad, Perú.

\section{MATERIALES Y MÉTODOS}

El estudio se desarrolló en la empresa Agroindustrial Casa Grande, en la provincia de Ascope, departamento de La Libertad. La zona se encuentra ubicada a una altitud de 234 msnm y presenta una temperatura ambiental entre 18 y $23^{\circ} \mathrm{C}$ y una humedad relativa de $75 \%$.

La muestra estuvo constituida por 45 yeguas de 2 a 4 años de edad con algunos signos de parasitosis y, que no hayan sido desparasitadas tres meses previos al estudio. Las yeguas se distribuyeron al azar en tres grupos: I: Control; II: antiparasitario doramectina; III: antiparasitario fenbendazole, con 15 repeticiones cada uno. Las unidades experimentales recibieron el mismo manejo, alimentación y condiciones ambientales durante la evaluación. Para la obtención de datos de cada unidad experimental, se obtuvieron muestras fecales y pruebas del parche antes y 30 días después (Sumano y Ocampo, 1997) de suministrados los tratamientos siguiendo la misma metodología.

Las muestras fecales se obtuvieron directamente del recto en las primeras horas de la mañana durante tres días con un día por medio (incrementa la sensibilidad de las pruebas de laboratorio) depositándolas en bolsas de plástico de primer uso, a estas muestras se adicionó bicromato de potasio al 2\% en proporción 1:1 como solución preservante o conservante (Pietrzak-Johnston et al., 2000; Domenech et al., 2009; INS, 2014). Finalmente se mezclaron las tres muestras fecales y se obtuvo una sola muestra por animal, que fue depositada en frascos de plástico de boca ancha de primer uso y mantenidas en refrigeración para su análisis. Tanto las muestras fecales como las pruebas del parche se enviaron al laboratorio antes de las 24 horas de haber sido obtenidas.

Los antiparasitarios fueron administrados por vía oral después de la obtención de la tercera muestra fecal pre tratamiento (quinto día) según grupos establecidos: I: control, no tratado II: antiparasitario doramectina $1.75 \%$, a razón de $11,4 \mathrm{mg} / \mathrm{kg}$ de peso vivo (jeringa de 6,84 $\mathrm{g}$ tiene 6 marcas, cada marca de producto es para 100 kg de pv.); III: antiparasitario fenbendazole 15\%, a razón de 7,5 mg/kg de peso vivo.

El análisis coproparasitológico se realizó en el Laboratorio de Helmintología Parasitaria del Departamento de Microbiología y Parasitología, Facultad de Ciencias Biologías, Universidad Nacional de Trujillo. Se analizó cualitativa y cuantitativamente, aplicando como alternativas diagnosticas las técnicas de kato Katz, para observar formas parasitarias (huevos) y cuantificar el número de huevos de nematodos por gramo de heces (hgh) respectivamente (Hendrix, 1999; Beltrán et al., 2014), Baermann modificado en copa para detectar huevos de nematodos (Thienpont et al., 1986; Hendrix, 1999; Beltrán et al., 2014) y, para la identificación de oxiuros se aplicó la técnica de Graham o prueba del parche en la región peri-anal y observadas al microscopio a 100X. Los parásitos fueron identificados a través de las características morfológicas de las formas parasitarias (huevos) (Hendrix, 1999; Sarmiento et al., 1999; Chaparro-Gutiérrez et al., 2018).

Para determinar el grado o intensidad del parasitismo pre y post tratamiento se utilizó el número de huevos por gramo de heces (nhpg), aplicándose la fórmula nhpgh = nho $\times$ factor, donde nho = número de huevos observados, y Factor $=24$. Los rangos del nhpg e interpretación de los resultados fueron:

$$
\begin{aligned}
& \text { - 100: sin parásitos }( \pm) \\
& \text { - } 101 \text { - 300: ligeramente parasitado }(+) \\
& \text { - } 301 \text { - 500: parasitado }(++) \\
& \text { - } 501 \text { - 700: muy parasitado }(+++) \\
& \text { - > 700: extremadamente parasitado }(++++)
\end{aligned}
$$


La eficacia de los antiparasitarios se determinó con el método del porcentaje de reducción de huevos de nematodos en la materia fecal por gramo de heces (hpg) mediante la fórmula descrita por Coles et al., (1992) y Vidyashankar et al., (2012): $E=[(c-t r) / c] \times 100$, donde $E=$ porcentaje de eficacia, $c=h p g$ antes del tratamiento, y $\mathrm{tr}=\mathrm{hpg}$ después del tratamiento.

Los resultados se expresaron en porcentaje asignándoles una valoración de acuerdo al siguiente criterio de evaluación (Rojas, 2004):

- Altamente eficaz: >98\%; Eficaz: 90-98\%; Ayuda en el control: 80-89\%; Insuficientemente activo o poco eficaz: $<80 \%$.

Para el análisis de datos se aplicó una prueba de normalidad, prueba de homogeneidad de varianza de Levene en pre tratamiento; análisis de varianza para comparar el número de huevos de nematodos gastrointestinales por gramo de heces (hpg) de los grupo en pre y post tratamiento y prueba de Duncan, estadística descriptiva para comparar y determinar, media y medidas de dispersión (rango y desviación estándar) pre y pos tratamiento, porcentaje y prueba de Chi cuadrado con un 95\% de confianza para determinar diferencias estadísticas de la eficacia entre tratamientos, utilizando el paquete estadístico SPSS Statistic v. 20.

\section{RESULTADOS Y DISCUSIÓN Nematodos gastrointestinales}

Todas las yeguas estuvieron parasitadas con nematodos previo al tratamiento (Tabla 1, Figura 1), lo cual concuerda con otros estudios que indican que el parasitismo gastrointestinal es una de las patologías más comunes en la especie equina (Nielsen, 2012; Reinemeyer, 2012; Von Samson-Himmelstjerna, 2012; Herrera et al., 2015; Salas-Romero et al., 2017; Chaparro-Gutiérrez et al. (2018).

\section{Tabla 1}

Frecuencia de especies parasitarias gastrointestinales previo al tratamiento antiparasitario en $\mathbf{1 5}$ muestras fecales por grupo de yeguas de Ascope, La Libertad (2013)

\begin{tabular}{lcccccc}
\hline Especie parásitaria & I. Control & \multicolumn{3}{c}{ II. Doramectina } & \multicolumn{2}{c}{ III. Fenbendazol } \\
& $\mathrm{n}^{\circ}$ & $\%$ & $\mathrm{n}^{\circ}$ & $\%$ & $\mathrm{n}^{\circ}$ & $\%$ \\
\hline Strongylus sp & 12 & 80 & 14 & 93.3 & 13 & 86.7 \\
Ascaris (Parascaris sp) & 10 & 66.7 & 7 & 46.7 & 9 & 60 \\
Oxyuris sp & 15 & 100 & 15 & 100 & 15 & 100 \\
\hline
\end{tabular}

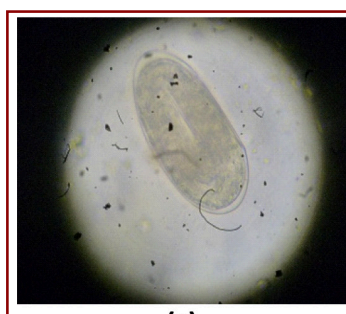

(a)

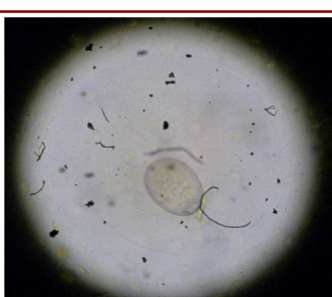

(b)

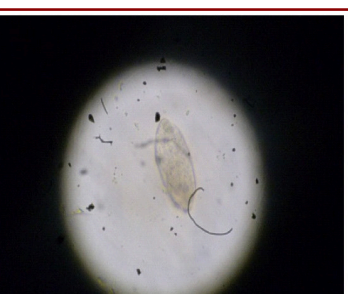

(c)

Figura 1. Huevos de nematodos gastrointestinales en yeguas de Ascope, La Libertad. (a) Huevo de Strongy/us. Transparentes, ovoides, 50-58 $\mu$ m de diámetro. (b) Huevo de Ascaris (Parascaris sp). Color parduzco, irregularmente esféricos de $90 \times 100 \mu \mathrm{m}$ de diámetro. Tiene una membrana gruesa y rugosa. (c) Huevo de Oxyuris. Asimétricos, casi siempre embrionados, 40×90 $\mu \mathrm{m}$. Se encuentran rodeados de una película pegajosa amarillenta 
Las especies Strongylus sp, Ascaris (Parascaris $\mathrm{sp)}$ y Oxyuris correspondieron a los nematodos gastrointestinales de mayor relevancia encontrados en el estudio, acorde con diversos estudios (Rodríguez-Vivas et al., 2001; Upjohn et al., 2010; Morales et al., 2012; Prokulewicz et al., 2014; Salas et al., 2014; Piccoli et al., 2015; Chaparro-Gutiérrez et al., 2018), aunque con variaciones en orden y porcentajes de frecuencia. Los Strongy/us pre tratamiento se encontraron en un mayor porcentaje de yeguas, después de los Oxyuris, concordante con Reinemeyer (2009) y Salas-Romero et al. (2017); sin embargo, Chaparro-Gutiérrez et al. (2018), encontraron valores menores para strongylus y oxyuris. Parascaris spse encontró en un menor porcentaje de animales, concordante con Francisco et al. (2012) y Peregrine et al., (2014). En el caso de Oxyuris sp, todas las yeguas resultaron positivas. Resultados semejantes encontraron otros estudios (Lyons y Oliver, 2004; Cardona et al., 2005;
Lyons et al., 2006). Cabe resaltar, que ChaparroGutiérrez et al., (2018) reportaron que el sexo y la edad de los equinos fueron factores de riesgo para strongylus y oxyuris, predominando estos en las hembras equinas mayores de un año de edad. Así mismo, cabe mencionar que Lester et al., (2013), consideran que un mayor número de animales parasitados y con niveles moderados de hpg, constituyen un riesgo, pues contaminan el ambiente y, por lo tanto, se predisponen a los animales a la infección o reinfección continua (Herrera et al., 2016).

En términos generales en la Tabla 2, se muestra la media y medidas de dispersión de los hpg de nematodos gastrointestinales en los tres grupos en pre y post tratamiento. Al análisis de datos en el pre tratamiento se aceptó la normalidad de los datos en los tres grupos y, a la prueba de homogeneidad de varianza de Levene se observó que los 3 grupos fueron homogéneos ( $p>0.05)$, cumpliéndose los requisitos de normalidad y homogeneidad de varianzas.

\section{Tabla 2}

\begin{tabular}{|c|c|c|c|c|c|}
\hline & \multirow{2}{*}{$\begin{array}{l}\text { Tratamiento } \\
\text { control }\end{array}$} & \multicolumn{2}{|c|}{ Pre tratamiento (día 0) } & \multicolumn{2}{|c|}{ Post tratamiento (día 30) } \\
\hline & & $\begin{array}{l}\text { Sin doramectina } \\
(n=15)\end{array}$ & $\begin{array}{l}\text { Sin fenbendazole } \\
(n=15)\end{array}$ & $\begin{array}{l}\text { Con doramectina } \\
(n=15)\end{array}$ & $\begin{array}{l}\text { Con fenbendazole } \\
(n=15)\end{array}$ \\
\hline & hpg & hpg & hpg & hpg & hpg \\
\hline Media & 595 & 597 & 621 & 18 & 291 \\
\hline $\mathrm{DE}$ & \pm 93.9 & \pm 104.2 & \pm 109.2 & \pm 23.1 & \pm 67.8 \\
\hline Min. & 456 & 432 & 408 & 0 & 120 \\
\hline Max. & 768 & 744 & 768 & 70 & 384 \\
\hline
\end{tabular}

Como se observa el número promedio de huevos de nematodos gastrointestinales por gramo de heces (hpg), en pre tratamiento fue similar en cada grupo ( $p>0.05)$. El rango de hpg antes de la dosificación varió entre 408 a 768 hpg. En base a estos valores en pre tratamiento, se considera que el grado o intensidad de parasitismo de las yeguas se encontró entre los tipos: parasitada $(++)$, muy parasitadas $(+++)$ y extremadamente parasitada $(++++)$.

Al respecto, identificar el tipo de parásitos y el grado de infección es de vital importancia clínica, ya que algunos parásitos son altamente patógenos (Molento et al., 2012). Canaver et al., (2013), refieren que dentro de la familia Strongylidae las poblaciones de grandes estróngilos vienen siendo reemplazadas por las de pequeños estróngilos y que a nivel mundial son el grupo más prevalente y patógeno (Kaplan, 2005, Matthews 2008). Esta tendencia se atribuye a la mayor capacidad de estos últimos de desarrollar resistencia a los antihelmínticos, en 
especial a los benzimidazoles (Scott et al., 2015; Salas-Romero et al., 2017).

En el post tratamiento el número promedio de huevos de nematodos gastrointestinales por gramo de heces (nhpg) de los grupos sin tratamiento, con doramectina y con fenbendazole difieren entre sí. El nhpg se redujo muy significativamente en el grupo que recibió doramectina $(p<0,01)$ y a la prueba de Duncan se encontró diferencias estadísticas ( $p$ $<0.05)$ entre grupos. En el grupo que recibió fenbendazole la reduccion del nhpg fue menor. Considerando el nhpg (291) post tratamiento y tomando en cuenta la clasificación establecida por Reinemeyer (2009), este grupo se constituiría en un grupo de riesgo de contaminación moderada (recuentos >200 y $<500$ hpg), contribuyendo a la contaminación ambiental con estados infectantes potenciales (Kaplan, 2005, Reinemeyer, 2009), ocasionando infección o reinfección de potrillos u otras categorías al permanecer en sistemas de alimentación en pastoreo, como lo refieren Matthews (2014);
Lester y Matthews, (2014) sugiriendo el control y tratamientos dirigidos para prevenir dicha contaminación.

\section{Eficacia de los antiparasitarios}

Según los resultados (Tabla 2 y 3), hay una significativa reducción del recuento de hpg a los 30 días de la dosificación con doramectina $1.75 \%$ en gel, mostrando una eficacia de $97 \%$ ( $p<0.01$ ) en el control de nematodos, mientras que el antiparasitario fenbendazole resultó poco eficaz (51\%) (Rojas, 2004). Los resultados para doramectina son similares con lo reportado por Cerna (2007), quien al utilizar doramectina oral encontró una eficacia sobre el 95\% hasta el día 35 post tratamiento, igualmente Tang et al., (2008), demostraron una efectividad antinematódica del 100\% a los 7 días del tratamiento en caballos usando un gel oral de doramectina. En forma similar, Pérez et al., (2010) indican eficacia vía oral hasta los 30 días post aplicación, mientras que Prokulewicz et al., (2014) evidenciaron 96$100 \%$ de eficacia en el control de nematodos gastrointestinales con este antiparasitario.

\section{Tabla 3}

Número de huevos de nematodos gastrointestinales por gramo de heces (hpg) previo a la dosificación (día $\mathbf{0}$ ) y a los $\mathbf{3 0}$ días de la dosificación y eficacia con doramectina y fenbendazole en yeguas de Ascope, La Libertad

\begin{tabular}{llll}
\hline & $\begin{array}{c}\text { Control } \\
(\mathrm{n}=15)\end{array}$ & Doramectina $(\mathrm{n}=15)$ & Fenbendazole $(\mathrm{n}=15)$ \\
\hline Día 0 & 595 & 597 & 621 \\
Día 30 & 559 & 18 & 291 \\
Eficacia (\%) & & 97 & 51 \\
\hline
\end{tabular}

Chávez et al., (2007), demostraron alta eficacia de doramectina $1.75 \%$ contra nematodos del género Strongylus en equinos hasta el día 63 y con capacidad efectiva hasta el día 70 post tratamiento; consideraron así mismo, que la efectividad hallada fue superior a otros estudios usando solo fenbendazole. Varady et al., (2004) reportaron eficacias del fenbendazole entre 84.4y 99.4\% en la reducción de huevos tipo Strongylus entre los 10 y 14 días posteriores a la dosificación y $100 \%$ de reduccion para huevos de $P$ equorum, mientras que Stratford et al., (2013), evidenciaron reducida eficacia del fenbendazole en Escocia.

Como se observó con el fenbendazole se obtuvo poca eficacia, al respecto hay evidencias de resistencia de los pequeños estrongylus a los productos a base de benzimidazoles, como lo reportaron Chávez et al., (2007). García et al., (2013) y Matthews (2014), indican que 
una mutación del gen de la beta-tubulina en nematodos de rumiantes y en varias especies comunes de ciatostomas les confiere la capacidad de resistencia a este grupo farmacológico. La resistencia a los compuestos antihelmínticos es un problema de preocupación mundial, que se da en la ganadería, particularmente en crianza bajo sistemas de pastoreo (Steffan et al., 2014); siendo la heredabilidad de la resistencia la característica más importante de este fenómeno (Kassai, 2002).

La estimación de la eficacia antihelmíntica mediante la comparación de recuentos de huevos en heces de animales antes y después de los tratamientos, es una forma importante de orientar los tratamientos y el antiparasitario de elección, así como, para detectar resistencia con la reducción de huevos, considerando resistencia al antiparasitario cuando la reduccion de hpg es menor al 95\% de efectividad como lo refiere Kassai (2002).

Temando en cuenta la farmacocinética, la tolerancia y su eficacia antihelmíntica, la administración vía oral de doramectina es una alternativa a los antiparasitarios convencionales para el tratamiento y control de las enfermedades parasitarias gastrointestinales de los equinos como lo consideran también Herrera et al., (2015).

\section{CONCLUSIONES}

El 100\% de las yeguas mostraron nematodiasis gastrointestinal, presentando alguna de las especies Strongylus sp, Pascaris sp y Oxyuris sp, predominando los huevos de Strongylus.

El antiparasitario de administración oral doramectina en gel, tuvo mayor eficacia (97\%) en el control de nematodos, mientras que el fenbendazole presentó una menor eficacia (51\%) en el control de nematodos gastrointestinales de la población en estudio.

Por el porcentaje de eficacia la doramectina en gel de administración oral sería el antiparasitario de elección para el control de nematodos gastrointestinales en equinos.

\section{REFERENCIAS BIBLIOGRAFICAS}

Beltran M, Tello CR, Náquira VC. (2014). Manual de procedimientos de laboratorio para el diagnóstico de los parásitos intestinales del hombre (Serie de Normas Técnicas 37). Lima, Perú: Instituto Nacional de Salud. 104 p.

Burn CC, Dennison TL, Whay HR. (2009). Environmental and demographic risk factors for poor welfare in working horses, donkeys and mules in developing countries. Vet J 186: 385-392. DOI: 10.1016/j.tvjl.2009.09.016.

Canaver RJ, Braga PRC, Boeckh A, Grycajuck M, Bier D, Molento MB. (2013). Lack of Cyathostomin sp. Reduction after anthelmintic treatment in horses in Brazil. Vet Parasitol; 194:35-39. DOl: https:// doi.org/10.1016/j.vetpar.2012.12.020

Cardona E, Choperena M, Quijano J, López G. (2005). Caracterización de nematodos gastrointestinales de equinos que llegan a la central ganadera de Medellín. Rev Cienc Pec 4: 384-385. Disponible en: https://www. redalyc.org/pdf/2950/295022975018.pdf

Cerna F. (2007). Estudio comparativo de la efectividad de dos lactonas macrocíclicas, administradas por vía oral e intramuscular en equinos. Tesis de pregrado. Chile. Universidad Austral de Chile. 28 p. Disponible en: http://cybertesis.uach.cl/ tesis/uach/2007/fvc415e/doc/fvc415e.pdf

Coles GC, Bauer C, Borgsteede FH, Geerts S, Klei TR, Taylor MA, Waller PJ. (1992). World Association for the Advancement of Veterinary Parasitology (W.A.A.V.P.) methods for the detection of anthelmintic resistance in nematodes of veterinary importance. Vet Parasitol 44:35-44. DOI: $\quad$ 10.1016/0304-4017(92)90141-U

Chaparro- Gutiérrez J, Ramírez-Vásquez N, Piedrahita D, Strauch A, Sánchez A, Tobón J, Olivera-Ángel M, Ortiz-Ortega D, ViLLarArgaiz D. (2018). Prevalencia de parásitos gastrointestinales en equinos y factores 
de riesgo asociados en varias zonas de Antioquia, Colombia. CES Medicina Veterinaria y Zootecnia, vol. 13, núm. 1, 2018. DOI: 10.21615/cesmvz.13.1.1.

Chávez A, Casas G, Casas E. (2007). Evaluación de la eficacia de un antiparasitario en solución conteniendo Triclabendazole, Ivermectina y Fenbendazole

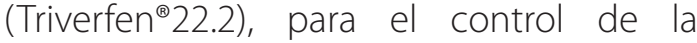
nematodiasis gastrointestinal en equinos. Perú. Disponible en: https://www.engormix. com/equinos/articulos/evaluacion-eficaciaantiparasitario-solucion-t27223.htm.

Chávez L. (2013). Doramectina oral para el tratamiento y control de parásitos internos en caballos. Disponible en: $\quad$ https://www.engormix.com/ equinos/articulos/doramectina-oraltratamiento-control-t30217.htm.

Doménech, C. I., Marcet, S. R., Figueredo, P. M., \& Sarracent, P. J. (2009). Conservación de heces humanas para la detección de antígenos de excreción-secreción de Fasciola hepatica. Rev. Cubana. Med. Trop., 61(2), 1-5. Disponible en: http:// scielo.sld.cu/pdf/mtr/v61n2/mtr14209.pdf

Francisco R, Paz-Silva A, Francisco I, Cortiñas $F$, Miguelez S, Suarez J, CazapalMonteiro C, Arias M, Sanchez R. (2012). Preliminary analysis of the results of selective therapy against Strongylus in pasturing horses. J Equine Vet Sci 32: 274-280. DOI: 10.1016/j.jevs.2011.09.074.

Garcia A, Brady HA, Nichols WT, Prien S. (2013). Equine cyathostomin resistance to fenbendazole in Texas horse facilities. J Equine Vet Sci 33: 223228. DOl: 10.1016/j.jevs.2012.06.005.

Gutiérrez SO, Gutiérrez FD. (2013). Teniasis en equinos de servicio público de la ciudad de Trinidad,Bolivia.RevCientAgroAmaz.1:43-47.

Hendrix CM. (1999). Diagnóstico Parasitológico
Veterinario. España: Harcourt-Brace.

Herrera Y, Perdomo S, Cardana J. (2015). Eficacia de la doramectina via intramuscular en nematodos gastrintestinales en equinos. RevMedVet29:41-49.DOI:10.19052/mv.3445.

Herrera Y, Vergara J, Ensuncho C, Causil L. (2016). Frecuencia de parásitos gastrointestinales en burros criollos (Equus africanus asinus) en el departamento de Córdoba, Colombia. Rev Colombi Cienc Anim 8:159166. DOI: 10.24188/recia.v8.n2.2016.183.

Herrera Benavides, Yonairo Manuel, Perdomo Ayola, Sandra Carolina, \& Cardona Álvarez, José Alberto. (2015). Eficacia de la doramectina vía intramuscular en nematodos gastrointestinales en equinos. Revista de Medicina Veterinaria, (29), 41-49. Disponible en: http://www.scielo.org.co/ scielo.php?script=sci_arttext\&pid=S012293542015000100005\&lng=en\&tlng=es.

Instituto Nacional de Salud (2014). Manual de procedimientos de laboratorio para el diagnóstico de los parásitos intestinales del hombre. Serie de Normas Técnicas 37. 104 pp. Lima- Peru. Disponible en: https://bvs.ins.gob.pe/ insprint/SALUD_PUBLICA/NOR_TEC/2014/ serie_normas_tecnicas_nro37.pdf.

Kaplan RM. (2005). Current concepts for parasite control in horses: it ain't the 60's anymore. Proceedings of the Kentucky Veterinary Medical Association, Morehead, Kentucky, USA, Pp 1-13.

Kassai, T. (2002). Helmintología Veterinaria, 1a Edición. Editorial Acribia S.A., Zaragoza-España. pp 4-13; 147-161.

Lester HE, Spanton J, Stratford CH, Bartley DJ, Morgan ER, Hodgkinson JE, Coumbe K, et al. (2013). Anthelmintic efficacy against cyathostomins in horses in Southern England. Vet Parasitol 197: 189196. DOI: 10.1016/j.vetpar.2013.06.009. 
Lester HE, Matthews JB. (2014). Faecal worm egg count analysis for targeting anthelmintic treatmentinhorses:pointstoconsider.Equine Vet J 46: 139-145. DOI: 10.1111/evj.12199.

Lyons ET, Tolliver SC. (2004). Prevalence of parasite eggs (Strongyloides westeri, Parascaris equorum, and Strongylus) and oocysts (Emeria leuckarti) in the feces of thoroughbred foals on 14 farms in central Kentucky in 2003. Vet Parasitol 5: 400404. DOl: 10.1007/s00436-003-1068-2.

Lyons ET, Tolliver SC, Collins SS. (2006). Field studies on endoparasites of thoroughbred foals on seven farms in central Kentucky. Vet Parasitol 98: 496-500.

MattoTN,BharkadGP,BhatSA.(2015).Prevalence of gastrointestinal helminth parasites of equids from organized farms of Mumbai and Pune. J Parasitic Dis 39: 179-185. DOI:

10.1007/s12639-013-0315-4.

Matthews JB. (2008). An update on cyathostomins: anthelmintic resistance and worm control. Equine Vet Educ 20: 552-560. DOI: 10.2746/095777308X363912.

Matthews JB. (2014). Anthelmintic resistance in equine nematodes. Int J Parasitol-Drug 4 310-315. DOI: 10.1016/j.jpddr.2014.10.003.

Molento MB, Kielsen MK, Kaplan RM. (2012). Resistance to avermectin/milbemycin anthelmintics in equine cyathostomins - Current situation. Vet Parasit; 185:1624. DOI: 10.1016 / j.vetpar.2011.10.013

Morales AA, Bello H, Vallejo M, Villoria D. (2012). Prevalencia de parásitos gastrointestinales en equinos Pura Sangre de Carrera durante el período decuarentena 2011 en el hipódromo "La Rinconada" Caracas, Venezuela. Rev Ibero-Latinoam Parasitol 6: 15-119. Nielsen M. (2012). Sustainable equine parasite control: perspectives and research needs. Vet Parasitol 185: 32-
44. DOl: $\quad 10.1016 /$ j.vetpar.2011.10.012.

Osterman E. (2005). Prevalence and control of Strongyle nematode infections of horses in Sweden. Doctoral Thesis. Uppsala, Sweden: Swedish University of Agricultural Sciences. 61 p.

Peregrine AS, Molento MB, Kaplan RM, Nielsen MK. (2014). Anthelmintic resistance in important parasites of horses: does it really matter? Vet Parasitol 201: 1-8. DOl: $\quad$ 10.1016/j.vetpar.2014.01.004

Pérez R, Godoy C, Palma C, Muñoz L, Arboix M, Alvinerie M. (2010). Plasma disposition and fecal elimination of doramectin after oral or intramuscular administration in horses. Vet Parasitol 170: 112-119. DOl: $\quad$ 10.1016/j.vetpar.2010.01.038.

Piccoli C, Marques SMT, Appel G, Silveira E, Siqueira GB, Loos DE, Mattos MJT. (2015). Helmintos intestinalis em cavalos de trabalho e de lazer de Porto Alegre/RS. Sci Anim Health 3:56-64. DOl: 10.15210/sah.v3i1.4227.

Pietrzak-Johnston SM, Bishop H, Wahlquist S, Moura H, De Oliveira Da Silva N, Pereira Da Silva S, Nguyen-Dinh P. (2020). Evaluation of commercially available preservatives for laboratory detection of helminths and protozoa in human fecal specimens. J Clin Microbiol. 38:1959-64. DOI: 10.1128 / JCM.38.5.1959-1964.2000

Prokulewicz A, Pilarczyk B, Tomza-Marciniak A. (2014). Evaluation of the efficacy of doramectin in the control of strongyle (strongylidae, cyathostominae) infestation in Horses. Isr J Vet Med 69: 83-87. Disponible en: http://www.ijvm. org.il/sites/default/files/pilarczyk.pdf

Proudman, C., \& Matthews, J. (2000). Control of intestinal parasites in horses. Equine Practice, 22, 90-97. DOl: https:// doi.org/10.1136/inpract.22.2.90 
Reinemeyer C. (2008). Parasite control for horses during the first year of life. In: Proc American Association of Equine Practitioners. Austin, Texas, USA.

Reinemeyer C.R. (2009). Controlling strongyle parasites of horses: a mandate for change. In: Proc55thAnnualConvention oftheAmerican Association of Equine Practitioners. Las Vegas, Nevada, USA. Pp 352-360. Disponible en: $\quad$ http://veterinaryextension.colostate. edu/menu2/equine/z9100109000352.pdf

Reinemeyer CR. (2012). Anthelmintic resistance in non-strongylid parasites of horses. Vet Parasitol 185: 9-15. DOl: $\quad$ 10.1016/j.vetpar.2011.10.009.

Rodríguez-Vivas R Ligia A. Cob-Galera, J. Domínguez-Alpizar. (2001). Frecuencia de parásitos gastrointestinales en animales domésticos diagnosticados en Yucatán, México. Revista Biomédica Vol. 12 N 1. DOl: doi.org/10.32776/revbiomed.v12i1.253.

Rojas M. (2004). Nosoparasitosis de los rumiantes domésticos peruanos. $2^{\circ}$ ed. Lima, Perú. Martegraf. 146 p.

Salas R, Mencho JD, Guerra L, Mencho JC. (2014). Prevalencia de nematodos intestinales y eficacia de Labiomec $^{\circledast}$ en caballos de Camagüey, Cuba. Rev Salud Anim 36: 152-158. Disponible en: http://scielo. sld.cu/pdf/rsa/v36n3/rsa03314.pdf

Salas-Romero J, Gómez-Cabrera K, Molento M, Lyon ET, Delgado A, González L, Arenal A, Nielsen M. (2017). Efficacy of two extra-label anthelmintic formulations against equine strongyles in Cuba. VetParasitol Reg Stud Rep 8: 39-42. DOI: 10.1016/j.vprsr.2017.01.010.

Salas-Romero J., Gómez K., Chicoy Y., Yero C., Valle E., Delgado-Martínez A., Salas-Romero J., Arenal A. (2017). Especies de ciatostomas resistentes al albendazol en equinos, Cuba. Rev Inv Vet Perú 28: 658-666. DOl: $\quad$ 10.15381/rivep.v28i3.13347.
Sarmiento L, Tantaleán M, Huiza A. (1999). Nematodos parásitos del hombre y de los animales en el Perú. Rev Peruana Parasit, 14(1-2): 9-65. Disponible en: https://sisbib. unmsm.edu.pe/BVRevistas/parasitologia/ v14_n 1-2/pdf/a02v14n1-2.pdf

Sumano L, Ocampo C. (1997). Farmacología veterinaria. 2a. ed. México: McGraw-Hill Interamericana.

Steffan, P., Fiel, C., Saumell, C., Fusé, C., Iglesias, L. (2014). El uso de antihelmínticos en los programas de control y el riesgo potencial de resistencia. Pp 85-94. Disponible en: http://helminto.inta. gob.ar/pdf\%20Resistencia/Steffan.pdf.

Stratford $\mathrm{CH}$, Lester HE, Pickles KJ, McGorum BC, Matthews JB. (2013). An investigation of anthelmintic efficacy against strongyles on equine yards in Scotland. Equine Vet J 46: 17-24. DOl: 10.1111/evj.12079.

Scott I, Bishop R, Pomroy W. (2015). Anthelmintic resistance in equine helminth parasites-a growing issue for horse owners and veterinarians in New Zealand? New Zeal Vet J 63: 188-198. DOl: $\quad 10.1080 / 00480169.2014 .987840$.

Tang J, Ledesma V. (2006). Evaluación de tolerancia y eficacia de un endectocida en gel sobre la base de doramectina (doraQuest I.A.) en el tratamiento de parasitosis internas de caballos de salto. Disponible en: http://www. agrovetmarket.com/pdf/antiparasitario/ DoraquestLA/Doraquest.pdf.

Tang J, Ruiz F, Rodriguez L. (2008). Evaluación de tolerancia y eficacia inmediata de un endectocida en gel sobre la base de doramectina al 1,75\% (doraQuest l.a.) en el tratamiento y control de parasitosis internas en equinos de la sierra central. Agrovetmarket. Disponible en: http:// www.doramectina.com/pdf/eficacia_ tolerancia_doramectina_evaluacion_ 
endectocida_doramectina_equinos.pdf.

Teixeira WF, Felippelli G, Cruz BC, Maciel WG, Fávero FC, Gomes LV, Buzzulini C, Prando L, Bichuette MA, Lopes WD, Oliveira GP, Costa AJ. (2014). Endoparasites of horses from the Formiga city, located in center-west region of the state of Minas Gerais, Brazil. Rev Bras Parasitol V 23: 534538. DOI: 10.1590/s1984-29612014072.

Thienpont D, Rochette F, Vanpaijs O.(1986). Diagnóstico de las helmintiasis por medio del examen coprológico. 2da ed.Bélgica: Janssen Research Fundation.

Upjohn MM, Shipton K, Lerotholi T, Attwood G, Verheyen KL. (2010). Coprological prevalence and intensity of helminth infection in working horses in Lesotho. Trop Anim Health Pro 42: 1655-1661. DOl: $\quad$ 10.1007/s11250-010-9617-z.

Varady M, Konigova A, Corba J. (2004). A field study to evaluate the efficacy of fenbendazole on 9 stud farms. Vet Med-Czech 49: 42-46. Disponible en: https://www.agriculturejournals. c z/publicFiles/61399.pdf

Velásquez C. (2012). Infestaciones parasitarias en los caballos. [Internet]. Disponible en: $\quad$ http://www.engormix.com/MAequinos/sanidad/articulos/infestacionesparasitarias-caballos-t4081/165-p0.htm.

Vidyashankar AN, Hanlon BM, Kaplan RM. (2012). Statistical and biological considerations in evaluating drug efficacy in equine strongyle parasites using fecal egg count data. Vet Parasitol 185: 4556. DOI: 10.1016/j.vetpar.2011.10.011.

Von Samson-Himmelstjerna G. (2012). Anthelmintic resistance in equine parasites detection, potential clinical relevance and implications for control. Vet Parasitol 185: 2-8. DOI: 10.1016/j.vetpar.2011.10.010. Wang, C.C. (2001). Basic Principles of
Antiparasitic Chemotherapy. In: Katzung B: Basic \& Clinic Pharmacology. P.869 881. 8a edition. Appleton \& Lange. USA. 